\title{
Efecto del tratamiento térmico sobre las concentraciones finales de calcio, fósforo y hierro en pitahaya amarilla (selenicereus megalanthus)
}

\author{
Junior Rodolfo López Engracia \\ juniorlopeze@hotmail.com \\ Facultad de Salud, Nutrición y Deporte \\ Universidad Internacional Iberoamericana. Campeche - México. \\ María José Rodriguez Andaluz \\ majoandaluz10@gmail.com \\ Facultad de Ciencias Agraria \\ Universidad Agraria Del Ecuador. Milagro - Ecuador.
}

\section{RESUMEN}

El objetivo de este estudio fue determinar cómo afecta la aplicación de tratamientos térmicos y utilizar cáscaras de pitahaya para cuantificar las concentraciones de calcio, fósforo y hierro en pulpa, harina y en pan. Se aplicó un tratamiento térmico mediante el uso de un deshidratador para obtener harina y posteriormente desarrollar un producto para consumo humano como lo es el pan. Se experimentaron 3 porcentajes diferentes de harina de pitahaya con respecto a la harina de trigo $(20 \%, 10 \%$ y $30 \%)$. Se utilizó un diseño completamente al azar de 3 repeticiones para cuantificar Fe, Ca y P, por medio de un espectrofotómetro de absorción atómica y de UV/visible. Como resultado los minerales presentes en la pulpa y en la cascara de la pitahaya se concentraron significativamente al igual que las cantidades de nutrientes después de pasar por un proceso térmico a los cual se les realizo un análisis de varianza y se compararon las medias con el Test de Tuckey $(\mathrm{p}<0,05)$. Los datos fueron tabulados por el programa estadísticos InfoStat. Se ha concluido que la pitahaya amarilla contiene un valor nutricional muy alto y puede ser utilizada como materia prima para el desarrollo de nuevos productos, incluso su cáscara se convierte en polvo, manteniendo intacto el contenido mineral.

Palabras clave: pitahaya amarilla; deshidratación adiabática; minerales; industrialización. 


\title{
Effect of thermal treatment on final concentrations of calcium, phosphorus and iron in yellow pitahaya (selenicereus megalanthus)
}

\begin{abstract}
The objective of this study was to determine how the application of heat treatments affects and use pitahaya shells to quantify the concentrations of calcium $(\mathrm{Ca})$, phosphorus $(\mathrm{P})$ and iron (Fe) in pulp, flour (adiabatic dehydration) and in bread. A thermal treatment was applied through the use of a dehydrator to obtain flour and subsequently develop a product for human consumption such as bread. Three different percentages of pitahaya flour were experimented with respect to wheat flour $(20 \%, 10 \%$ and $30 \%)$. A completely randomized design of 3 repetitions was used to quantify $\mathrm{Fe}, \mathrm{Ca}$ and $\mathrm{P}$, by means of an atomic absorption and UV/visible spectrophotometer. As a result, the minerals present in the pulp and in the shell of the pitahaya were significantly concentrated, as well as the amounts of nutrients after going through a thermal process, to which an analysis of variance was carried out and the means were compared with the Test Tuckey $(\mathrm{p}<0.05)$. The data was tabulated by the statistical program InfoStat. It has been concluded that the yellow pitahaya contains a very high nutritional value and can be used as raw material for the development of new products, even its shell turns into powder, keeping the mineral content intact.
\end{abstract}

Keywords: yellow pitahaya; adiabatic dehydration; minerals; industrialization.

Artículo recibido: 02 enero 2022 Aceptado para publicación: 28 enero 2022 Correspondencia: juniorlopeze@ hotmail.com Conflictos de Interés: Ninguna que declarar 


\section{INTRODUCCIÓN}

Actualmente en Ecuador se está produciendo la Pitahaya de variedad amarilla tipo 1, que presenta diferencias, principalmente en el tamaño según el lugar del cultivo, cabe indicar que algunos datos señalan que la Pitahaya amarilla representa alrededor del $90 \%$ de lo producido en el país.

La pitahaya es rica en fibra, esta se encuentra principalmente en las semillas, las cuales al ser ingeridas se adhieren a las vellosidades intestinales y ayudan a movimiento peristáltico de las heces, es decir hacen que estas no se queden retenidas en los intestinos, sino que salgan (Rivera, 2014).

Esta fruta posee cualidades como tónico general por la cual se recomienda para mejorar la circulación y la prevención de las enfermedades del corazón, es ligeramente sedante que se utiliza para algunos dolores leves como el dolor de la cabeza y el dolor de las encías, combate la ansiedad, el insomnio y el estreñimiento regulando la flora intestinal de las personas (Vera, 2011).

Las propiedades de esta fruta generan amplias ventajas, por este motivo tiene un alto grado de aceptación en el mercado y para su producción se requiere de trabajo e inversión, esta fruta produce en zonas subtropicales y amazónicas del Ecuador.

La pitahaya es una fruta exótica con varios beneficios positivos para la salud, es por eso que en los mercados internacionales existe una demanda positiva de esta fruta. Para saber si la fruta está en buen estado y lista para consumir, se lo debe observar el color, es mejor si esta amarilla. La pitahaya contiene las vitaminas A, B1, B2, B3 y C, respecto a los minerales no aportan calcio, hierro y fosforo (Zabala, 2005).

Las vainas de la pitahaya son de color amarillo intenso, con espinas color cremoso, su peso oscila entre 250 gramos y alcanza hasta 19 grados Brix, la cáscara es de color amarillo y su pulpa blanca al madurar. La pitahaya amarilla tiene un mayor interés comercial y agronómico en el mercado local como internacional, ya que tiene mayor resistencia al transporte y almacenamiento, su sabor es más agradable.

El calcio es esencial para el crecimiento y formación de huesos, manteniendo su fortaleza y su densidad. Se encuentra ligado como agente de prevención de enfermedades relacionadas con el sistema nervioso, coagulación de la sangre, osteoporosis y contracción de músculos (Miller et. al 2013).

El fósforo es importante para el metabolismo humano. Entre el 80-90\% de fósforo del 
organismo se combina para formar fosfato cálcico, que es utilizado para el desarrollo de huesos y dientes, al igual que el calcio. Los huesos son la mayor reserva de sales de fosfato en el organismo (McClure et al., 2017).

El hierro es un mineral que está relacionado con el crecimiento y desarrollo físico y mental de niños y adultos. Ayuda a transportar oxígeno a los tejidos y mantiene el sistema inmunológico en buen estado, aumentando las defensas del organismo y generando una mayor resistencia a las enfermedades (Rebellato, 2015).

La deshidratación es una de las formas más antiguas de conservar alimentos y tiene como objetivo eliminar gran parte de la humedad de los productos. Los alimentos deshidratados mantienen gran proporción de su valor nutricional si el proceso se realiza de forma adecuada (Krokida y Philippopoulos, 2005).

Las harinas en la industria alimentaria representan una importante materia prima para la obtención de muchos alimentos, y generalmente son de cebada, maíz o trigo. Su proceso consiste en la molienda del grano, pero en la actualidad se están utilizando frutas deshidratadas molidas como alternativa a las harinas convencionales (Uthumporn et al., 2015).

El propósito de esta investigación es cuantificar calcio, fósforo y hierro en dos procesos térmicos aplicados a la pitahaya y en su pulpa desarrollar harina y un producto listo para consumo, y determinar la cantidad de minerales si se mantiene estable, disminuye o aumenta

\section{ESTRATEGIAS METODOLÓGICAS O MATERIALES Y MÉTODOS}

La materia prima utilizada para la deshidratación se utilizó la pitahaya amarilla (Selenicereus megalanthus). La fruta seleccionada es la que reflejaba estado íntegro, firmeza y sin presencia de daños físicos ni microbiológicos.

Se lavaron y desinfectaron las frutas de la pitahaya amarilla por inmersión en una solución de dióxido de cloro líquido a $10 \mathrm{ppm}$ durante 10 minutos como lo propone Pao et al. (2017). Con esta proporción se consigue una reducción logarítmica sobre las unidades formadoras de colonia que pueden estar presentes en frutas y hortalizas. Los frutos se pelaron donde se separaron en dos partes la pulpa y corteza, ambas partes fueron utilizada en la investigación.

Se aplicaron $61 \pm 3^{\circ} \mathrm{C}$ hasta tener una pérdida de humedad mínima de $85 \%$, valores que establece la norma técnica ecuatoriana para harina de trigo (INEN 616:2015). 
Se realizaron mediciones cada 30 minutos para determinar la evolución de secado. La pérdida de humedad se calculó bajo las recomendaciones de la norma mexicana Cuantificación de hierro, calcio y fósforo en procesos térmicos aplicados a la pitahaya (Selenicereus megalanthus). 277 NMX (F-083-1986) y representó en porcentaje de humedad. Posteriormente la materia prima pasó por un proceso de molienda, pulverizado y tamizado para obtener la harina.

En la investigación de Álzate et al. (2013) y Giani et al. (2016) indica que el análisis de concentración de hierro, calcio y fosforo en harina de frutas es la caracterización más importante que se puede realizar por su estabilidad, posible incremento en los procesos de secado y aporte nutricional. Con esta referencia se utilizó un diseño completamente al azar de 3 repeticiones para cada tratamiento y así cuantificar hierro, calcio y fosforo en la pulpa y en el producto deshidratado por medio de un espectrómetro de absorción atómica UV/visible. Se utilizaron los métodos AOAC927.02 (Fe), 945.03 (Ca) y $965.17(\mathrm{P})$ para verificar como varia la cantidad de micronutrientes en el producto después de ser expuesto al tratamiento térmico.

Además de conocer si es viable elaborar harina de buena composición a partir de una fruta no tradicional, se efectuaron mezclas de harina de trigo en conjunto con la metería prima deshidratada para obtener un producto de repostería y tener la opinión de los catadores, con el fin de evaluar su aceptación en el mercado.

\section{RESULTADOS Y DISCUSIÓN}

\section{Deshidratación}

Se deshidrato la cascara de la pitahaya amarilla para obtener la harina propuesta, teniendo una humedad de (+/- 0,5) porciento, la correlación del tiempo de secado (9 horas y 8 horas) por aire caliente. Tal como en la investigación de Akonor et al. (2012) se logró el mismo porcentaje $(+/-0,5)$ de humedad en la harina obtenida, con un $8,45 \%$ y $7,33 \%$ para cada muestra.

Los resultados de esta sección se equiparán en parte a los logrados en el trabajo realizado por Castillo et al. (2012), donde se obtuvo harina a partir del nopal con un porcentaje de humedad después de la deshidratación de $6 \%$, pero el tiempo requerido para elaborar la harina fue de 24 horas con una temperatura de $70^{\circ} \mathrm{C}$.

\section{Parámetro de control}

Los resultados de la medición de $\mathrm{pH}$ y grados Brix realizada a la muestra de harina obtenida 
de la cascara de la pitahaya es de 3,8 de pH y 7\% de sólidos solubles. Los valores de $\mathrm{pH}$ difieren de la investigación realizada por Santos et al. (2014) en el desarrollo de harina de dos variedades de papaya, donde obtuvieron un valor de 4,5 para el $\mathrm{pH}$, pero se asemeja al valor conseguido en grados Brix con $6 \%$.

\section{Cuantificación de minerales (hierro, calcio y fósforo)}

En la tabla 1 se observan los resultados de la cuantificación de minerales en la pulpa y harina de Pitahaya los resultados obtenidos después del proceso térmico aumentan significativamente en relación con su peso inicial: Fosforo y Calcio supera la ingesta diaria recomendada (IDR), mientras que los valores de Hierro están por debajo de la IDR. Estos valores de referencia se encuentran en el artículo realizado por la Federación Española de Sociedades de Nutrición, Alimentación y Dietética (2010), donde se señala que la recomendación para hierro en adultos es de 18 mg/día, para calcio es de 900 mg/día y para fósforo es de $700 \mathrm{mg} /$ día. La cantidad cuantificada en pulpa y harina está representada en miligramos por cada 100 gramos de muestra.

Tabla 1. Valores medios de calcio, hierro y fósforo en pulpa, harina y en un producto a base de harina de pitahaya.

\begin{tabular}{cccc}
\hline Producto & $\begin{array}{c}\text { Calcio } \\
\mathbf{m g} / \mathbf{1 0 0 g}\end{array}$ & $\begin{array}{c}\text { Hierro } \\
\mathbf{m g} / \mathbf{1 0 0 g}\end{array}$ & $\begin{array}{c}\text { Fósforo } \\
\mathbf{m g} / \mathbf{1 0 0 g}\end{array}$ \\
\hline Pulpa de Pitahaya & $18,08 \mathrm{c} \pm 1,83$ & $8,11 \mathrm{c} \pm 1,93$ & $26,00 \mathrm{c} \pm 2,02$ \\
Harina de Pitahaya & $43,42 \mathrm{~b} \pm 1,31$ & $16,47 \mathrm{~b} \pm 2,33$ & $52,53 \mathrm{~b} \pm 1,25$ \\
Producto a base de Harina & $53,532 \mathrm{a} \pm 1,12$ & $30,73 \mathrm{a} \pm 2,53$ & $63,87 \mathrm{a} \pm 2,09$ \\
Trigo + pitahaya & & & \\
\hline
\end{tabular}

Las medias que no comparten una letra son significativamente diferentes para la Prueba de Tukey $(\mathrm{p}<0,05)$. n (5).

El contenido de minerales aumentó después de deshidratar la pulpa. Esto sucede porque los minerales, dependiendo de la materia prima, pueden tener un incremento durante distintos procesos tecnológicos, entre ellos los tratamientos térmicos (Quinteros, 2002). Los resultados de hierro, calcio y fósforo en la pulpa de pitahaya se pueden comparar con la investigación de Morais et al. (2017), donde se analizaron minerales en pulpa de banano, papaya, sandía y maracuyá, consiguiendo resultados en Ca superiores y en Fe inferiores. Los resultados también se pueden comparar con los valores de la investigación de Pizzani etal. (2008), donde se cuantificaron minerales en la harina de pijiguao (fruto del Amazonas). La cantidad de $\mathrm{Ca}$ $(43 \mathrm{mg} / 100 \mathrm{~g})$ y $\mathrm{P}(39 \mathrm{mg} / 100 \mathrm{~g})$ tiene un valor cercano a la harina de pitahaya mientras que el 
valor de Fe (1,56 mg/100g) es inferior al obtenido en el presente estudio.

\section{Análisis microbiológico}

En la tabla 2 se muestran los resultados microbiológicos obtenidos en la harina de Pitahaya, así como en los estudios realizados por Benítez et al. (2011), Ferreira et al. (2015) y Vedia et al. (2016), donde no se encontró contaminación en el desarrollo de harinas de fruta y vegetales. El deshidratado de la cascara de la pitahaya no presentó ningún valor por encima de los requisitos requeridos. El desarrollo del producto fue realizado bajo parámetros de calidad e higiene. Si la materia prima no presenta contaminación se puede innovar y desarrollar nuevos productos alimenticios.

Tabla 2. Análisis microbiológico en harina de Pitahaya

\begin{tabular}{cccc}
\hline Parámetros & Resultados & Requisitos & Método \\
\hline Aerobios Mesófilos & $63.4 \times 101 \mathrm{UFC} / g$ & $10.000 \mathrm{UFC} / g$ & NTE INEN 1529-5 \\
Mohos & $490 \mathrm{UFC} / \mathrm{g}$ & Máx.1000 UFC/g & NTE INEN 1529-10 \\
Levaduras & $870 \mathrm{UFC} / \mathrm{g}$ & Máx.1000 UFC/g & NTE INEN 1529-10 \\
Coliformes Totales & Ausente/g & Ausencia & NOM-093-SSA1-1994 \\
Escherichia Coli & $<10 \mathrm{UFC} / g$ & $<10 \mathrm{UFC} / g$ & ISO 16649-2
\end{tabular}

\section{Evaluación sensorial de producto con harina de Pitahaya}

La tabla 3 muestra los porcentajes de harina de pitahaya y de trigo utilizados para el desarrollo de un producto de repostería.

Tabla 3. Composición de producto a base de harina de pitahaya

\begin{tabular}{cccc}
\hline Materias & No1 (80/20) & No2 $(\mathbf{9 0 / 1 0})$ & No3 (70/30) \\
\hline Harina de trigo & $160 \mathrm{~g}$ & $180 \mathrm{~g}$ & $140 \mathrm{~g}$ \\
Harina de pitahaya & $40 \mathrm{~g}$ & $20 \mathrm{~g}$ & $60 \mathrm{~g}$ \\
\hline
\end{tabular}

El tratamiento $\mathrm{N}^{\circ} 1$ con $80 \%$ de harina de trigo y $20 \%$ de harina de la cascara de pitahaya tuvo la mejor aceptabilidad.

Este resultado lo podemos validar con el análisis de componentes principales (Ca, P y Fe) también realizados en la investigación de Villarroel et al. (2003), donde mediante el mismo método demostró que hubo un incremento en la concentración de Calcio y Fósforo, indicando de esta manera cuál de las formulaciones de galleta era la más idónea de acuerdo a un análisis sensorial.

Los resultados microbiológicos de la harina de pitahaya se encuentran dentro de los parámetros requeridos bajo las normas INEN (8518 1980-12), INEN (616:2015) 
establecidas en el Ecuador, para conteo de Aerofilos, Levaduras, Mohos, Coliformes Totales y Escherichia Coli teniendo similitud con lo reportado por López-Campos et al. (2009) quien trabajó en la obtención de harina de pitahaya roja. La cual luego fue suplementada con polifenoles para la alimentación a corderos, dando como resultado una mejor comunidad bacteriana ruminal.

Figura 1. Resultado de análisis sensorial "sabor"

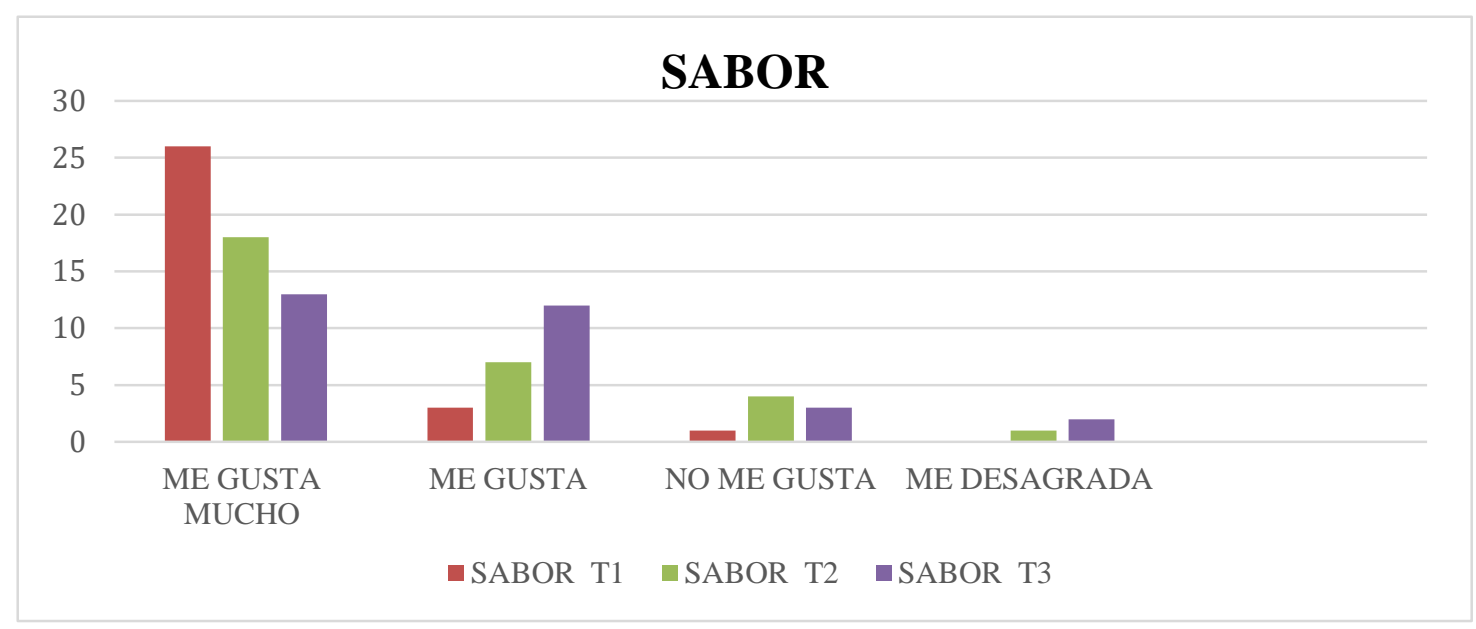

Podemos determinar que el atributo sabor correspondiente al tratamiento 1 tiene diferencias significativas con respecto a T2 y $\mathrm{T} 3$.

Figura 2. Resultado de análisis sensorial "color"

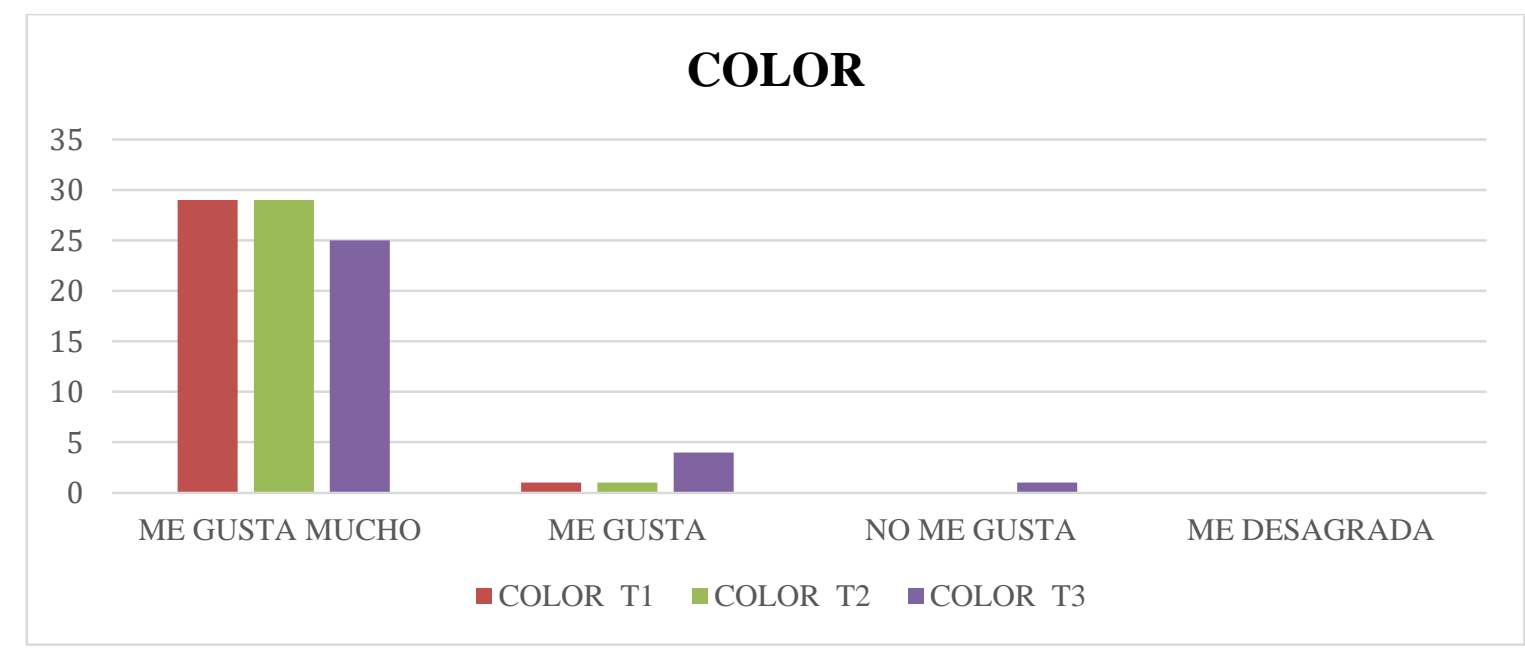

En la figura 2 se encuentran los resultados del análisis sensorial en este caso color. Los resultados para atributo color no existen diferencias significativas entre T1 y T2, pero con valores superiores con respecto a $\mathrm{T} 3$. 
Figura 3. Resultado de análisis sensorial "olor"

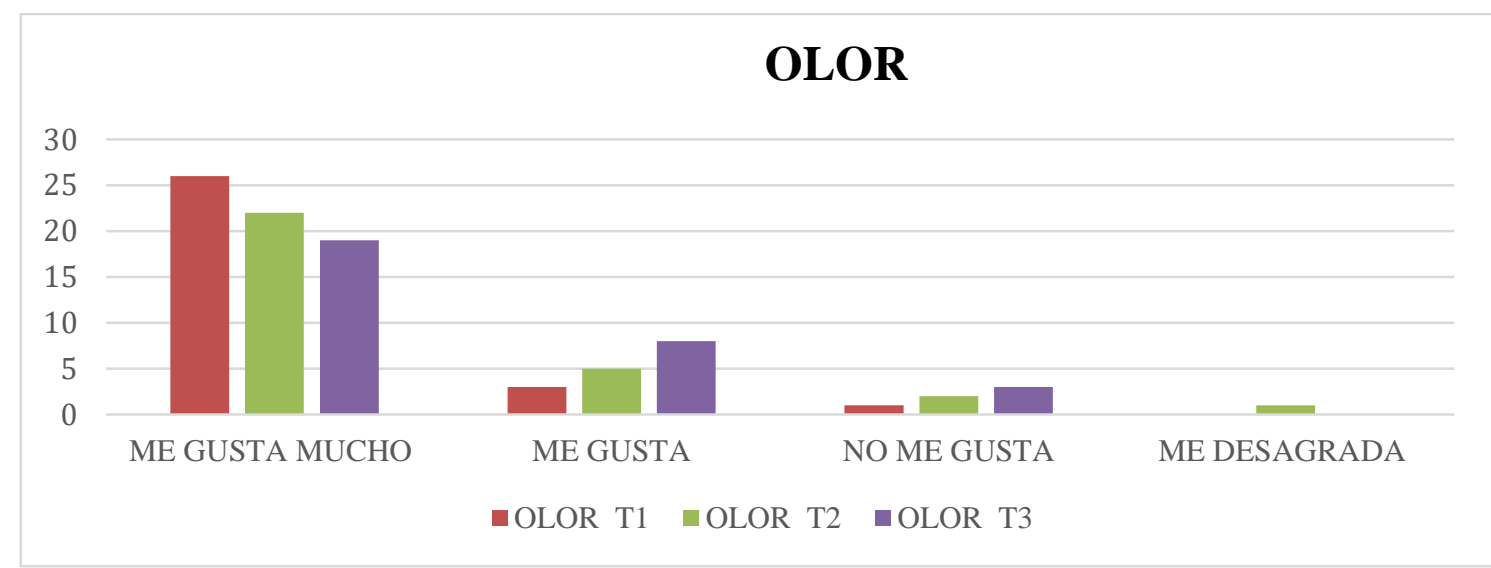

El atributo olor del T1 muestra un valor ligeramente superior a T2 y T3. En la figura 3 se encuentran los resultados del análisis sensorial en este caso olor.

. Figura 3. Resultado de análisis sensorial "textura"

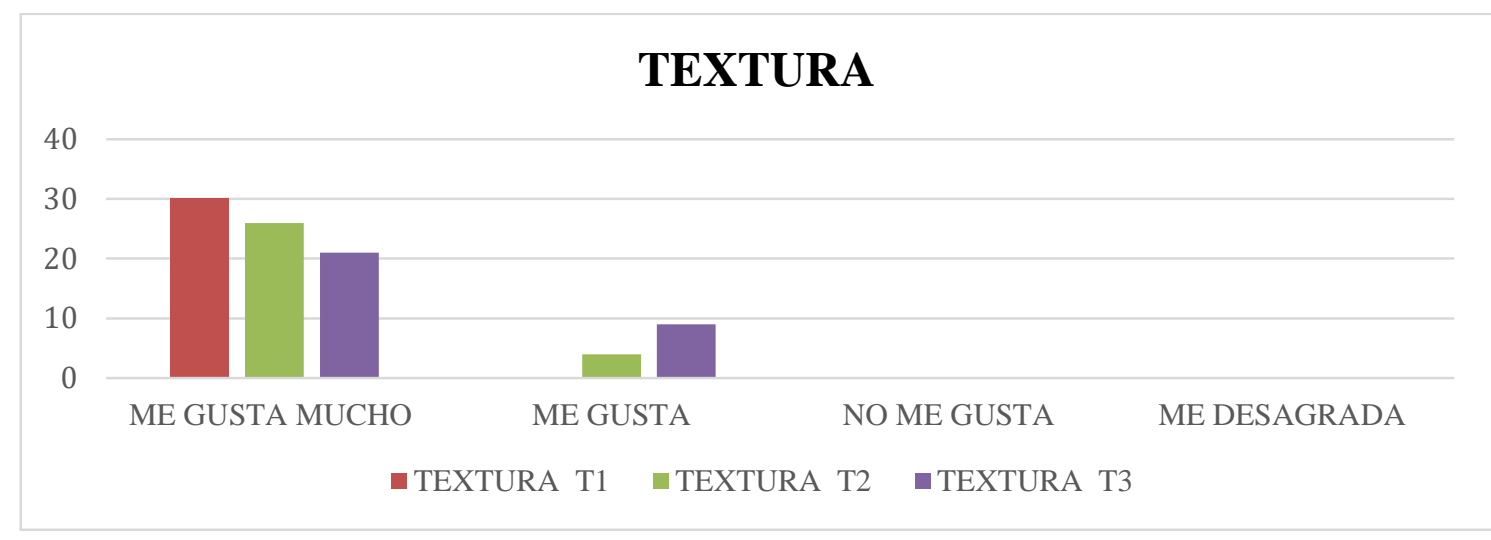

En la figura 4 se encuentran los resultados del análisis sensorial en este caso textura.

La ponderación del atributo textura del T1, tiene diferencias significativas con respecto a T2 y T3, por lo que es elegido como el tratamiento mejor evaluado en todos los atributos sensoriales. Los valores son similares a lo obtenido en el desarrollo de tortillas de maíz y soya elaboradas a partir de harina elaborado por Figueroa et al. (2011), donde no existe diferencia significativa.

\section{CONCLUSIÓN O CONSIDERACIONES FINALES}

Se demostró que el Hierro presente en la pulpa, en la harina y en un producto de repostería de pitahaya se encuentra por encima de la dosis diaria recomendada, lo que permite tener una materia prima que puede ayudar a suplir la carencia de este mineral en la dieta de las personas. 
Es necesario realizar un estudio de biodisponibilidad de hierro (no-hemo) de la fruta de pitahaya y analizar si su absorción puede ser influenciada por factores dietéticos y fisiológicos.

Los minerales, a diferencia de las vitaminas, son más estables a distintos procesos térmicos. En algunos casos, como en la presente investigación, no solo se pierden, si no que se concentran y aumentan significativamente su porcentaje.

\section{LISTA DE REFERENCIAS}

Akonor, P.; C. Tortoe; E. Buckman y L. Hagan. 2017. Proximate composition and sensory evaluation of root and tuber composite flour noodles. Cogent Food \& Agriculture, $3(1): 1-7$.

Álzate, E.: V Quintero y J. Lucas. 2013.

Determinación de las propiedades térmicas y composicionales de la harina y almidón de chachafruto (Erytina Edulis Triana Ex Micheli). Temas Agrarios, 18 (2): 21-35.

Benítez, B.; K. Ferrer; A. Archile; Y. Barboza; L. Rangel.; E. Márquez y M. Delmonte. 2011. Calidad microbiológica de una galleta formulada a base de harina de yuca y plasma de bovino. Revista de la Facultad de Agronomía, 28 (2): 260-272.

Castillo, S.; L. Estrada; M. Margalef y S. Toffoli. 2013. Obtención de harina de nopal y formulación de alfajores de alto contenido en fibra. Diaeta, 31 (142): 20-26.

Federación Española de Sociedades de Nutrición, Alimentación y Dietética. 2010. Ingestas dietéticas de referencia (IDR) para la población española, 2010. Actividad Dietética, 14 (4): 196-197.

Ferreira, M.; M. Santos; T. Moro; G. Basto.; R. Andrade y É. Gonçalves. 2015. Formulation and characterization of functional foods based on fruit and vegetable residue flour. Journal of Food Science and Technology, 52 (2): 822-830.

Figueroa, J.; M. Acero; N. Vasco; A. Lozano; L. Flores y F. Gonzalez-Hernandez.

Giani, C.; A. Vanderlei y M. Ornella. 2106. Prospecting the physical, chemical and nutritional characteristics of comercial fruit flours.Ntririon \& Food Sciene Intenational Journal, 2 (1): 555-577. Giraldo, C.

Krokida, M y C. Philippopoulos. 2005. Rehydration of Dehydrated Foods. Drying Technology, 23 (4): 799-830. DOI: 10.1081/DRT200054201.

McClure, S.; A. Chang; E. Selvin; C. Rebholz y L. Appel. 2017. Dietary Sources of Phosphorus among Adults in the United States: Results from NHANES 2001- 
2014. Nutrients, 9 (95): 2-10. DOI: 10.3390/nu9020095.

Miller, G.; J. Jarvis y L. McBean. 2001. The importance of meeting calcium needs with foods. Journal of the American College of Nutrition, 20 (2): 168-185. DOI: 10.1080/07315724.2001.10719029.

Morais, D.; E. Rotta; S. Sargi; E. Bonafe; R. Suzuki.; N. Souza; M. Matsushita y J. Visentainer. 2017. Proximate composition, mineral contents and fatty acid composition of the different parts and dried peels of tropical fruits cultivated in Brazil. Journal of the Brazilian Chemical Society, 28 (2): 308-318. DOI: 10.5935/0103-5053.20160178.

Pao, S.; D. Kelsy; M. Khalid y M. Ettinger M. 2007. Using aqueous chlorine dioxide to prevent contamination of tomatoes with Salmonella enterica and erwinia carotovora during fruit washing. Journal of Food Protection, 70 (3): 629-634. DOI:10.4315/0362-028X-70.3.629.

Pizzani, P.; M. Blanco; T. Malaver.; S. Godoy; I. Matute; J. Palma y N. Obispo. 2008. Composición fitoquímica y nutricional de harina de pijiguao (Bactris gassipaes Kunth en HBK). Zootecnia Tropical, 26 (3), 235-238.

Quinteros, A. 2002. Contenidos de calcio, magnesio, hierro, cinc y fósforo en legumbres crudas y sometidas a distintos procesos de cocción. Revista Amazónica de Investigación Alimentaria, 2 (1): 97-102.

Rebellato, A.; B. Pacheco; J. Prado y J. Lima. 2015. Iron in fortified biscuits: A simple method for its quantification, bioaccessibility study and physicochemical quality. Food Research International, 77 (3): 385-391. DOI: 10.1016/j.foodres.2015.09.028.

Rivera Camino, J. (2014). Marketing sectorial principios y aplicaciones. Madrid.

Santos, C.; Abreu; J. Freire; E, Queiroz y M. Mendoca. 2014. Chemical vharacterization of the flour of peel and seed from two papaya cultivarse. Food Science and Tecnology, 34 (2): 353-357.

Uthumporn, U.; W. Woo; A. Tajul y A. Fazilah. 2015. Physico-chemical and nutritional evaluation of cookies with different levels of eggplant flour substitution. CyTA Journal of Food, 13 (2): 220-226.

Vedia, V.; P. Gurak; S. Espinoza y J. Ruano. 2016. Calidad fisicoquímica, microbiológica y sensorial de tallarines producidos con sustitución parcial de sémola de trigo por 
harina de amaranto. Revista Española de Nutrición Humana y Dietética, 20 (3): 190-197.

Vera, J. (2011). pitahaya una alternativa para producción de frutas. El Agro.

Villarroel, L. J. Álvarez y D. Maldonado. 2003. Aplicación del análisis de componentes principales en el desarrollo de productos. Acta Nova, 2 (3) 399-408, 399-410.

Zabala Salazar, H. (2005). Planeación estratégica aplicada a cooperativas y demás formas asociativas y solidarias (Primera ed.). Colombia: Universidad Cooperativa de Colombia. 